\title{
Clinico-epidemiology of neuroblastoma in north east Egypt: A 5-year multicenter study
}

\author{
YOUSSEF AL-TONBARY ${ }^{1}$, MOHAMED BADR ${ }^{2}$, AHMED MANSOUR $^{1}$, USAMA EL SAFY $^{2}$, SHEBL SAEED $^{3}$, \\ TAMER HASSAN $^{2}$, RASHA ELASHERY ${ }^{1}$, ROFIDA NOFAL ${ }^{2}$ and AHMAD DARWISH ${ }^{1}$ \\ ${ }^{1}$ Department of Pediatric Hematology and Oncology, Mansoura University Children's Hospital, Mansoura, Dakahlia 35516; \\ ${ }^{2}$ Department of Pediatric Hematology and Oncology, Zagazig University Children's Hospital, Zagazig, Sharqia 44111; \\ ${ }^{3}$ Department of Pediatric Hematology and Oncology, Tanta University Children's Hospital, Tanta, Gharbia 31111, Egypt
}

Received July 16, 2014; Accepted February 22, 2015

DOI: $10.3892 / 01.2015 .3335$

\begin{abstract}
Neuroblastoma, an embryonal malignancy of the sympathetic nervous system, is the most frequent extracranial solid tumor The clinico-epidemiological features of neuroblastoma in infants and children were investigated between January 2005 and January 2010 at the Pediatric Oncology units of Mansoura, Zagazig, and Tanta University Children's Hospitals (Egypt). Of 142 cases of neuroblastoma, 10 were omitted from the study due to defective data. The median age of the patients was 30 months, with $75.8 \%$ aged $\geq 1$ year and $24.2 \%$ aged $<1$ year at time of diagnosis. The male-to-female ratio was 1.06. Suprarenal glands were the most common primary tumor site $(72.7 \%)$. The majority of the patients $(76.7 \%)$ had stage IV disease. Favorable pathology was observed in $43.8 \%$ of patients, while $56.2 \%$ exhibited unfavorable pathology. The estimated survival rate of patients was $30.7 \pm 10.0 \%$, and mean survival time was $24.2 \pm 5.2$ months. The rate of mortality was $28.6 \%$ for patients aged $<1$ year, and $81.8 \%$ for those aged $\geq 1$ year $(\mathrm{P}=0.005)$. For patients with favorable pathology, the rate of mortality was significantly lower $(28.6 \%)$ compared with that of patients with unfavorable pathology $(77.8 \%$; $\mathrm{P}=0.049$ ). Although the association between outcome and each of the primary tumor sites, children's oncology group risk and gender was statistically insignificant, a large effect size was identified between outcome and primary tumor site, as well as children's oncology group risk and a medium effect size was identified between outcome and gender. Additionally, an age of $\geq 1$ year was associated with unfavorable pathology $(\mathrm{P}=0.024)$, stage IV disease $(\mathrm{P}=0.026)$ and a suprarenal primary tumor site $(\mathrm{P}=0.001)$.
\end{abstract}

Correspondence to: Dr Tamer Hassan, Department of Pediatric Hematology and Oncology, Zagazig University Children's Hospital, 1 Zagazig University Street, Zagazig, Sharqia 44111, Egypt

E-mail: dr.tamerhassan@yahoo.com

Key words: epidemiology, neuroblastoma

\section{Introduction}

Neuroblastoma is an embryonal malignancy of the sympathetic nervous system that is derived from primordial neural crest cells. It is the most frequently occurring extracranial solid tumor, accounting for $9-10 \%$ of all pediatric neoplasms, and heavily contributes to childhood cancer mortality (1). The majority of cases are sporadic, and may occur as a localized or disseminated disease. Spontaneous regression may also be observed (2).

As the understanding of neuroblastoma tumors has increased, it has become evident that these tumors behave differently with regard to aggressiveness and response to treatment. Multimodal therapies are necessary to improve the prognosis of patients with progressive tumors, and aggressive therapy must be avoided in patients with favorable tumor pathology in order to reduce side effects and financial expenditure (3).

The Children's Oncology Group (COG) classifies neuroblastoma patients into low, intermediate and high risk categories based on certain characteristics including the age of the patient, the stage of the disease upon initial diagnosis, the histological appearance of the tumor, the quantitative DNA content of the tumor (ploidy), and the presence or absence of amplification of the MYCN oncogene (4).

The aim of the present study was to investigate the clinicoepidemiological features of neuroblastoma in infants and children in north east Egypt and to evaluate the association between these clinico-epidemiological features and patient outcome.

\section{Materials and methods}

The present retrospective study included 142 patients with neuroblastoma, who were diagnosed, treated and followed-up at four main hospitals (Mansoura University Children's Hospital and Mansoura Oncology Center, Mansoura; Zagazig University Hospital, Zagazig; and Tanta University Hospital, Tanta, Egypt) between January 2005 and January 2010. Of these cases, 10 were omitted from the study due to defective data records. The remaining 132 cases were analyzed for demographic characteristics, and factors impacting survival were determined. The 
clinical, morphological and biological data obtained for each patient were filed in a computerized database using SPSS software, version 14 (SPSS Inc., Chicago, IL, USA). A descriptive analysis was performed for each variable, and the associations between variables, and between each variable and outcome, were analyzed using the appropriate statistical method ( $\chi^{2}, \phi$, Cramer's V, Mann-Whitney, Kendall's $\tau_{\mathrm{B}}$ and Median tests). The effect sizes were estimated and used to evaluate the practical significance of the results. Survival was measured from the date of diagnosis to the date of death or of last follow-up. Overall survivals (OAS) were estimated using Kaplan-Meier curves and compared using a log-rank test and multivariate analysis using the Cox regression method. $\mathrm{P}<0.05$ was considered to indicate a statistically significant difference.

The study was undertaken in accordance with ethical standards and with the Helsinki Declaration of 1964 (as revised in 2000) and was approved by the ethical committees of Mansoura University, Zagazig University and Tanta University. Written informed consent was obtained from the patient's families.

\section{Results}

In total, 142 cases of neuroblastoma were identified from the hospital records between January 2005 and January 2010, and 10 cases were subsequently excluded due to defective data records. Of the remaining 132 cases, 28 were diagnosed in 2005, 36 in 2006, 44 in 2007, 8 in 2008 and 16 in 2009. The cohort comprised 68 males and 64 females; 32 patients were aged $<1$ year, and 100 were aged $\geq 1$ year. The median age at the time of diagnosis was 30 months (range, 2-96 months; Table I).

Among the 32 patients aged $<1$ year, the primary tumor site was suprarenal in 16 cases $(50.0 \%)$, cervical in 4 cases $(12.5 \%)$, paraspinal in 4 cases $(12.5 \%)$ and retroperitoneal in 8 cases $(25.0 \%)$. Of the 96 cases of patients aged $\geq 1$ year and with a known primary tumor site, $80(83.3 \%)$ were suprarenal, 4 (4.2\%) were paraspinal and $12(12.5 \%)$ were retroperitoneal; a significantly high proportion of patients aged $\geq 1$ year had suprarenal tumor sites $(\mathrm{P}=0.001, \mathrm{t}=27.250$, $\mathrm{df}=2, \chi^{2}$ non-parametric test for distribution within cases). No significant association was observed between age at diagnosis and site of the primary tumor $(\mathrm{P}=0.161, \phi$ test $)$ (Table II).

As International Neuroblastoma Staging System (INSS) stage IVS disease is defined by the age of the patient, cases of this stage were excluded from the statistical assessment of the association between age and tumor stage, in order to avoid bias. The correlation between age in months and disease stage was determined to be positive and significant $\left[\mathrm{r}=0.265, \mathrm{P}=0.045\right.$ (one-tailed), Kendall's $\tau_{\mathrm{B}}$ test $]$. In addition, a significant association between age (ranked into $<1$ year and $\geq 1$ year) and stage was identified $[\mathrm{r}=0.356 ; \mathrm{P}=0.026$ (one-tailed), Kendall's $\tau_{\mathrm{B}}$ test; Table II].

A significant positive correlation was identified between age in months and Shimada pathological status $[\mathrm{r}=0.364$, $\mathrm{P}=0.050$ (one-tailed), Kendall's $\tau_{\mathrm{B}}$ test]; with an age cutoff of 1 year, a stronger correlation was identified, which was significant at the 0.01 level $[\mathrm{r}=0.618, \mathrm{P}=0.008$ (one-tailed), Kendall's $\tau_{\mathrm{B}}$ test; Table II].
Table I. Demographic characteristics of the neuroblastoma patients included in the study.

\begin{tabular}{lrr}
\hline Demographic characteristics & $\mathrm{n}$ & $\%$ \\
\hline Year & & \\
2005 & 28 & 21.2 \\
2006 & 36 & 27.3 \\
2007 & 44 & 33.3 \\
2008 & 8 & 6.1 \\
2009 & 16 & 12.1 \\
Age, years & & \\
$<1$ & 32 & 24.2 \\
$\geq 1$ & 100 & 75.8 \\
Gender & & \\
Male & 68 & 51.5 \\
Female & 64 & 48.5 \\
\hline
\end{tabular}

Among the 28 patients with favorable pathology, 4 (14.3\%) had stage II disease, 8 (28.6\%) had stage III and $16(57.1 \%)$ had stage IV. Of the 36 patients with unfavorable pathology, 8 patients $(22.2 \%)$ had stage III disease, $24(66.7 \%)$ had stage IV and $4(11.1 \%)$ had stage IVS. Progression in stage was associated with a higher proportion of unfavorable pathology cases, which reached $60.0 \%$ in stage IV tumors (Table III).

No significant association was found between pathological status and stage $(\mathrm{P}=0.535)$. The correlation between Shimada pathological status and stage according to INSS (by ranking them into non-distant metastatic stages (I, II, III) and metastatic stages (IV, IVS) was positive $(\mathrm{r}=0.221)$, but showed no statistical significance ( $\mathrm{P}=0.196$; Tables III and IV). Notably, a higher percentage of patients in the unfavorable pathology group exhibited evidence of central nervous system metastasis $(85.7 \%)$ when compared with the favorable pathology group $(55.6 \%)$.

Clinical presentations were variable, with abdominal mass being the most common ( $87.9 \%$; 116 cases), followed by pallor (57.6\%; 76 cases), fever (45.5\%; 60 cases), proptosis and/or raccoon eye $(42.4 \%$; 56 cases), bone pain $(36.4 \%$; 48 cases), skull masses (33.3\%; 44 cases), lower limb weakness $(30.3 \%$; 40 cases), abdominal pain $(15.2 \% ; 20$ cases), weight loss $(12.1 \%$; 16 cases $)$, urine retention $(9.1 \% ; 12$ cases $)$, constipation $(9.1 \% ; 12$ cases), diarrhea $(9.1 \% ; 12$ cases), purpura and/or bleeding $(6.1 \%$; 8 cases $)$, convulsions $(6.1 \% ; 8$ cases $)$ and facial palsy (3\%; 4 cases) (Fig. 1).

Of the 120 patients with known stage according to INSS, 8 cases $(6.1 \%)$ were stage IIB, 16 cases $(12.1 \%)$ stage III, 92 cases $(69.7 \%)$ stage IV and 4 cases $(3 \%)$ were stage IVS (Fig. 2).

Among the 116 patients with known risk according to COG risk group assignment for neuroblastoma, 8 cases $(6.1 \%)$ were of low risk, 8 cases $(6.1 \%)$ were of intermediate risk and 100 cases $(75.8 \%)$ were of high risk. The high risk group included 4 patients who were initially of low risk and were disease-free for a mean period of 18 months, but subsequently suffered recurrence in the form of bone secondary malignancies and bone marrow infiltration, and were 
Table II. Distribution of sites of the primary tumor, INSS and pathology by age at presentation.

\begin{tabular}{|c|c|c|c|c|c|}
\hline & \multicolumn{2}{|c|}{ Age, years } & \multirow[b]{2}{*}{ Test } & \multirow[b]{2}{*}{ Statistic } & \multirow[b]{2}{*}{ P-value } \\
\hline & $<1$ & $\geq 1$ & & & \\
\hline Site of primary tumor, n $(\%)$ & & & $\phi$ test & 0.401 & 0.161 \\
\hline Supra renal & $16(50.0)$ & $80(83.3)$ & & & \\
\hline Neck & $4(12.5)$ & $0(0.0)$ & & & \\
\hline Paraspinal & $4(12.5)$ & $4(4.2)$ & & & \\
\hline Retroperitoneal & $8(25.0)$ & $12(12.5)$ & & & \\
\hline INSS, n (\%) & & & Kendall's $\tau_{\mathrm{B}}$ & 0.359 & 0.026 \\
\hline IIB & $4(16.7)$ & $4(4.3)$ & & & \\
\hline III & $8(33.3)$ & $8(8.7)$ & & & \\
\hline IV & $12(50.0)$ & $80(87.0)$ & & & \\
\hline Pathology (Shimada classification), n (\%) & & & Kendall's $\tau_{B}$ & 0.618 & $0.008^{\mathrm{a}}$ \\
\hline Favorable & $20(83.0)$ & $8(20.0)$ & & & \\
\hline Unfavorable & $4(17.0)$ & $32(80.0)$ & & & \\
\hline
\end{tabular}

${ }^{\mathrm{a} O n e-t a i l e d . ~ I N S S, ~ I n t e r n a t i o n a l ~ N e u r o b l a s t o m a ~ S t a g i n g ~ S y s t e m . ~}$

Table III. Relationship between Shimada pathological status and stage according to INSS of the studied neuroblastoma cases. Valid cases, $n=64(48.5 \%)$.

\begin{tabular}{|c|c|c|c|c|c|c|c|}
\hline \multirow{2}{*}{$\begin{array}{l}\text { Pathology (Shimada } \\
\text { classification), n (\%) }\end{array}$} & \multicolumn{4}{|c|}{ INSS } & \multirow[b]{2}{*}{ Total } & \multirow[b]{2}{*}{ Statistic $^{a}$} & \multirow[b]{2}{*}{ P-value } \\
\hline & IVS & IIB & III & IV & & & \\
\hline Favorable & $0(0.0)$ & $4(14.3)$ & 8 (28.6) & $16(57.1)$ & $28(100)$ & 0.369 & 0.535 \\
\hline Unfavorable & $4(11.1)$ & $0(0.0)$ & $8(22.2)$ & $24(66.7)$ & $36(100)$ & & \\
\hline Total & $4(6.3)$ & $4(6.3)$ & $16(25.0)$ & $40(62.5)$ & $64(100)$ & & \\
\hline
\end{tabular}

${ }^{a}$ Cramer's V test. INSS, International Neuroblastoma Staging System.

Table IV. Association between Shimada pathological status and metastasis in the studied neuroblastoma cases. Valid cases, $64(48.5 \%)$.

\begin{tabular}{lccrr}
\hline & \multicolumn{3}{c}{ Metastasis } \\
\cline { 2 - 4 } $\begin{array}{l}\text { Pathology (Shimada } \\
\text { classification), n (\%) }\end{array}$ & $\begin{array}{l}\text { Non-metastatic } \\
\text { stages (I, II, III) }\end{array}$ & $\begin{array}{c}\text { Metastatic } \\
\text { stages (IV, IVS) }\end{array}$ & Total & Statistic $^{\text {a }}$ \\
\hline $\begin{array}{l}\text { Favorable } \\
\text { Unfavorable }\end{array}$ & $12(42.9)$ & $16(57.1)$ & $28(100)$ & 0.221 \\
$\begin{array}{l}\text { Total } \\
8(22.2)\end{array}$ & $28(77.8)$ & $36(100)$ & 0.196 \\
\hline
\end{tabular}

${ }^{\mathrm{a}}$ Kendall's $\tau_{\mathrm{B}}$ test.

reclassified as high risk, receiving the COG high risk treatment protocol (5) (Fig. 3).

The clinical, morphological and biological features of patients with known outcome, their distribution and bivariate analysis for outcome are listed in Table V. Of the 116 cases with known outcome, 64 were males, of which 28 (43.8\%) were alive at the end of the current study and $36(56.2 \%)$ succumbed to the disease. Of the 52 female patients, $8(15.4 \%)$ are alive, and $44(84.6 \%)$ succumbed to the disease. The gender of the patients and the clinical outcome were not significantly associated $(\mathrm{P}=0.101, \phi$ test $)$. However, a medium effect size was estimated for these variables $(r=0.305)$, and the likelihood of 


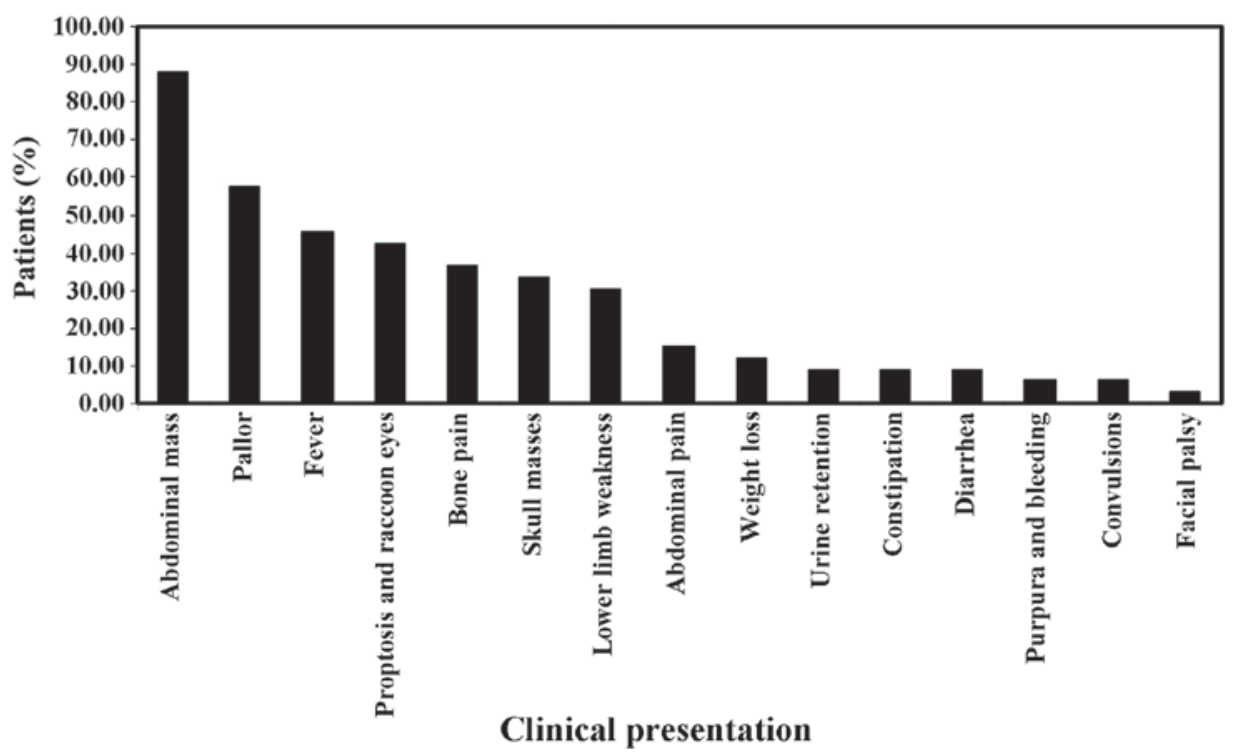

Figure 1. Clinical presentations of neuroblastoma patients. Abdominal mass, pallor and fever were the most common clinical presentations.

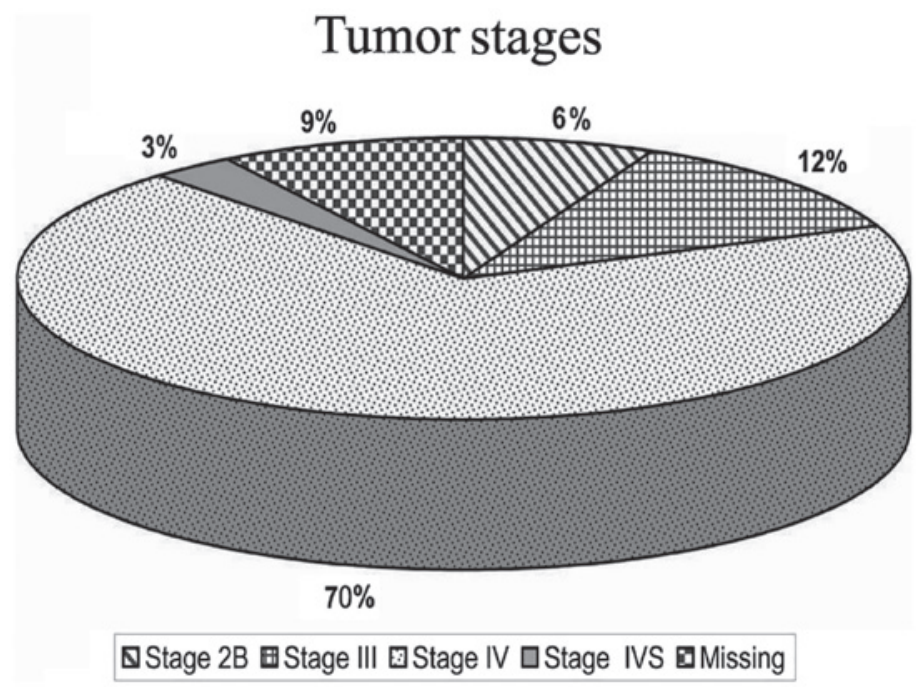

Figure 2. International Neuroblastoma Staging System stages of patients in the current study. The majority of the patients were classified as stage IV.

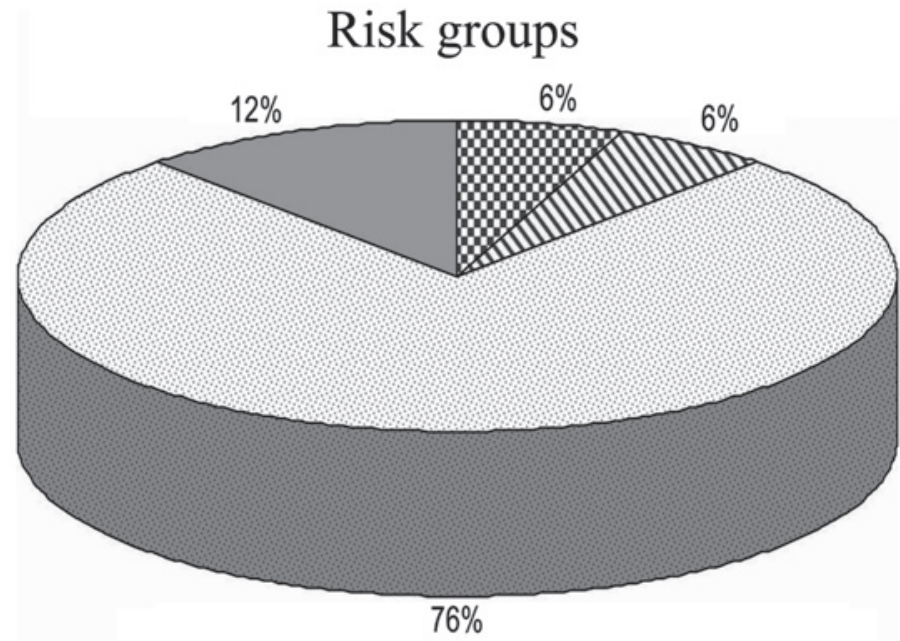

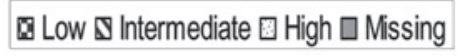

Figure 3. Children Oncology Group risk classification of neuroblastoma patients. The majority of the studied patients were classified as high risk. 


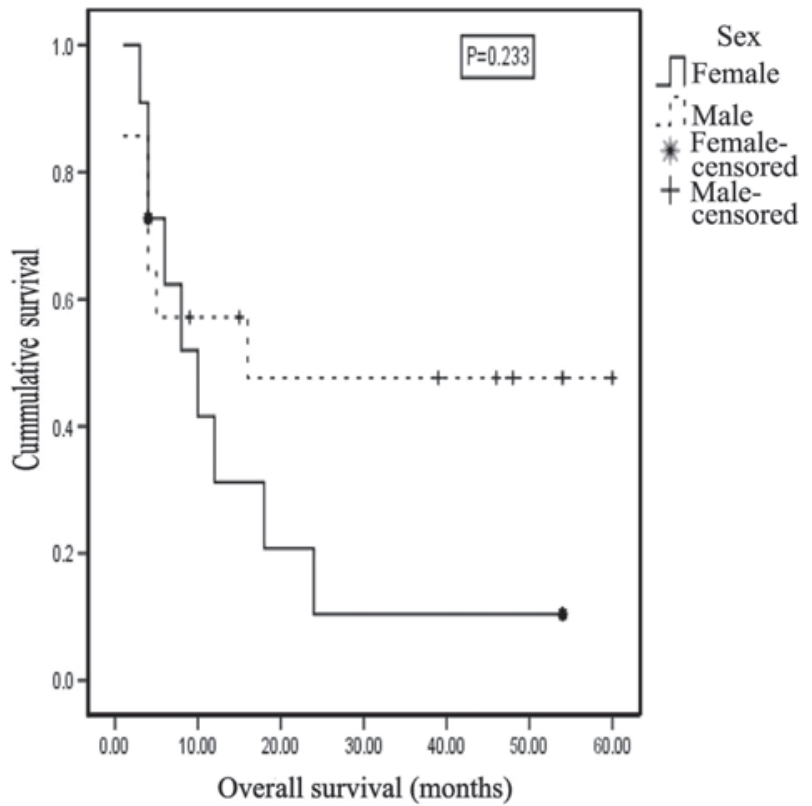

Figure 4. Kaplan Meier curve showing survival probability by gender for neuroblastoma patients. The 2 -year overall survival for males was $47.6 \pm 14.0 \%$, in comparison to $10.4 \pm 9.8 \%$ for females.

mortality among females was 4.9 times higher compared with that among males. The 2 -year OAS for males was $47.6 \pm 14.0 \%$ [mean \pm standard deviation (SD)], compared with $10.4 \pm 9.8 \%$ for females, with a mean survival time of $31.452 \pm 7.588$ months for males and $14.714 \pm 4.712$ months for females. However, this difference was not statistically significant ( $\mathrm{P}=0.233$ ) (Fig. 4).

A significant negative correlation was identified between age (in months) at presentation and outcome $[\mathrm{r}=-0.269$, with a medium effect size identified; $\mathrm{P}=0.047$ (one-tailed), Kendall's $\tau_{\mathrm{B}}$ test]. Furthermore, the correlation was stronger and significant at the $\mathrm{P}<0.01$ level (one-tailed) when an age cutoff of 12 months was applied ( $r=-0.493$ with a large effect size; $\mathrm{P}=0.005$, Kendall's $\tau_{\mathrm{B}}$ test). Among patients with known outcome, 88 were aged $\geq 1$ year, of which $18.2 \%$ survived and $81.8 \%$ died ( $\mathrm{P}=0.003, \chi^{2}$ test for non-parametric distribution). The median age at presentation was 10 months for survivors and 33 months for non-survivors. The odds of mortality among patients aged $\geq 1$ year was 9 times higher compared with that of patients aged $<1$ year. The 2 -year OAS for patients $<1$ year of age upon presentation was $71.4 \pm 17.1 \%$, while for patients $\geq 1$ year of age the OAS was $18.3 \pm 9.5 \%$, with a mean survival time of $40 \pm 8.4$ months for the former group and 17.6 \pm 5.1 months for the latter group. This difference was not statistically significant $(\mathrm{P}=0.061$, test statistic $=3.509, \log$ rank test; Fig. 5).

A significant association was identified between Shimada pathological status and outcome $(\mathrm{P}=0.049, \phi$ test $)$ and the effect size estimate indicated a large effect $(\mathrm{r}=0.492)$. The 2 -year OAS for the favorable pathology group was $71.4 \pm 17.1 \%$, compared with $19.0 \pm 16.8 \%$ for the unfavorable pathology group, with a mean survival time of $39.7 \pm 8.5$ months for the former and $17.7 \pm 8.9$ months for the latter; this difference was not statistically significant $(\mathrm{P}=1.36$, test statistic $=2.221, \log$ rank test; Fig. 6). The odds of mortality among cases with unfavorable pathology was 5.8 times higher compared with those of favorable pathology.

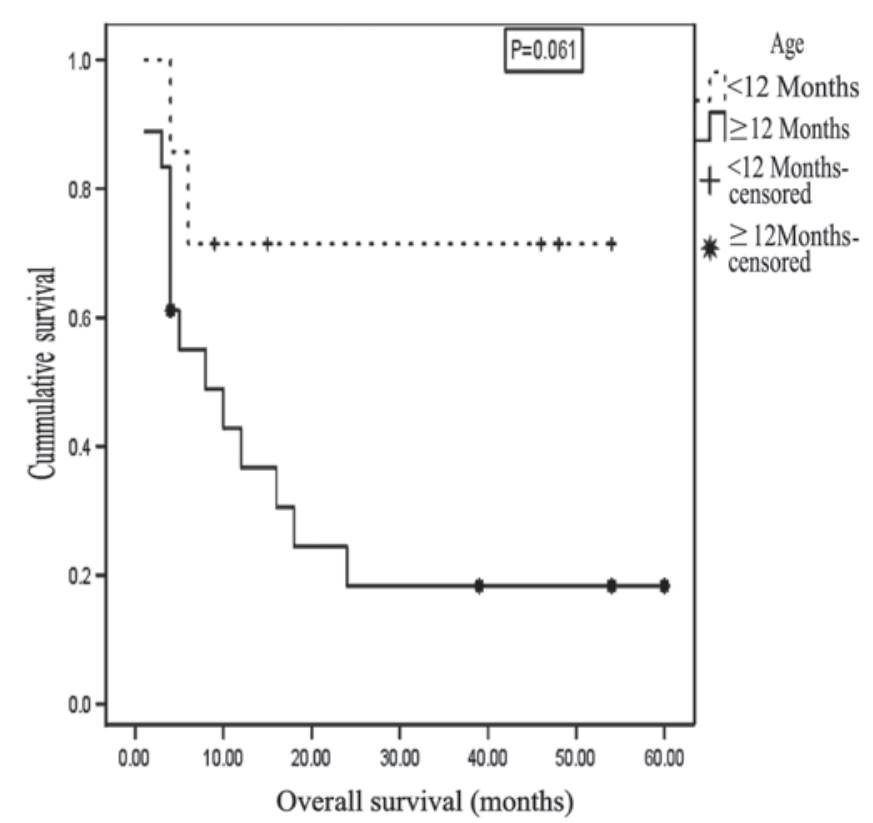

Figure 5. Kaplan-Meier curve showing survival probability by age for neuroblastoma patients. The 2 -year overall survival for patients aged $<1$ year at presentation was $71.4 \pm 17.1 \%$ while that of patients $\geq 1$ year was $18.3 \pm 9.5 \%$.

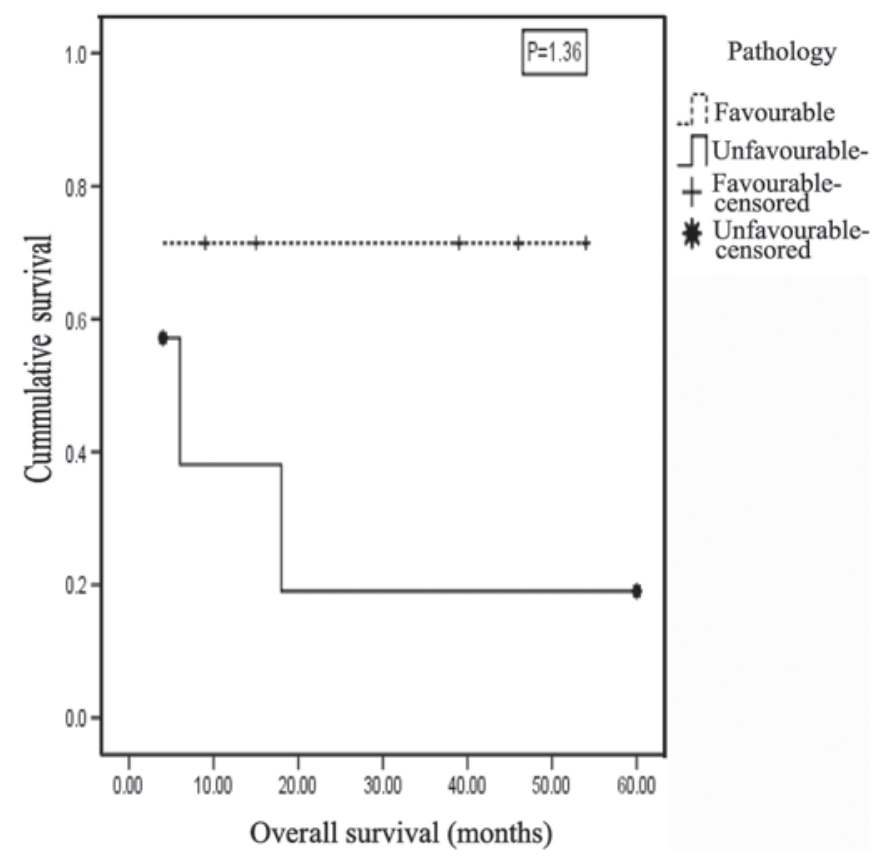

Figure 6. Kaplan-Meier curve showing survival probability by pathology for neuroblastoma patients. The 2-year overall survival for the favorable pathology group was $71.4 \pm 17.1 \%$, in comparison to $19.0 \pm 16.8 \%$ for the unfavorable pathology group.

The site of the primary tumor was not significantly associated with outcome $(\mathrm{P}=0.187$, Cramer's V test). However, a large effect size was identified ( $\mathrm{r}=0.414)$. The 2-year OAS for patients with suprarenal mass did not differ significantly from those with retroperitoneal mass $(27.8 \pm 10.6 \%$ vs. $75.0 \pm 21.7 \%$, respectively; $\mathrm{P}=0.449$, test statistic $=2.648, \log$ rank test).

In addition, no significant association was observed between INSS stage and outcome ( $\mathrm{P}=0.659$, Cramer's V test; $\mathrm{r}=0.235$, small effect size) or between risk stratification and 
Table V. Distribution of outcome by clinical, morphological and biological features.

\begin{tabular}{|c|c|c|c|c|c|c|c|}
\hline Characteristics & $\mathrm{n}$ & Valid, \% & Alive & Deceased & Statistic & P-value & Test \\
\hline Gender, n (\%) & & & & & & 0.101 & $\phi$ test \\
\hline Male & 64 & 51.5 & $28(43.8)$ & $36(56.2)$ & & & \\
\hline Female & 52 & 48.5 & $8(15.4)$ & $44(84.6)$ & & & \\
\hline Age, n (\%) & & & & & 0.493 & 0.005 & Kendall's $\tau_{B}$ \\
\hline$<1$ year & 28 & 24.2 & $20(71.4)$ & $8(28.6)$ & & & \\
\hline$\geq 1$ year & 88 & 75.8 & $16(18.2)$ & $72(81.8)$ & & & \\
\hline Primary tumor site, $\mathrm{n}(\%)$ & & & & & 0.414 & 0.187 & Cramer's V \\
\hline Suprarenal & 84 & 75.0 & $20(23.8)$ & $64(76.2)$ & & & \\
\hline Retroperitoneal & 24 & 21.4 & $16(66.7)$ & $8(33.3)$ & & & \\
\hline Cervical & 4 & 3.6 & $0(0.0)$ & $4(100.0)$ & & & \\
\hline INSS, n (\%) & & & & & 0.235 & 0.659 & Cramer's V \\
\hline II & 8 & 6.9 & $4(50.0)$ & $4(50.0)$ & & & \\
\hline III & 16 & 13.8 & $8(50.0)$ & $8(50.0)$ & & & \\
\hline IV & 88 & 75.9 & $24(27.3)$ & $64(72.7)$ & & & \\
\hline IVS & 4 & 3.4 & $0(0.0)$ & $4(100.0)$ & & & \\
\hline Shimada, n (\%) & & & & & 0.492 & 0.049 & $\phi$ test \\
\hline Favorable & 28 & 43.8 & $20(71.4)$ & $8(28.6)$ & & & \\
\hline Unfavorable & 36 & 56.2 & $8(22.2)$ & $28(77.8)$ & & & \\
\hline COG risk, n (\%) & & & & & 0.448 & 0.067 & Cramer's V \\
\hline Low & 4 & 3.7 & $0(0.0)$ & $4(100.0)$ & & & \\
\hline Intermediate & 8 & 7.4 & $8(100.0)$ & $0(0.0)$ & & & \\
\hline High & 96 & 88.9 & $24(25.0)$ & $72(75.0)$ & & & \\
\hline
\end{tabular}

INSS, International Neuroblastoma Staging System; COG, Children Oncology Group.

outcome ( $\mathrm{P}=0.067$, Cramer's V test); however, a large effect size was estimated for the latter $(r=0.448)$.

\section{Discussion}

In the present study, the male-to-female ratio was 1.06 , in concordance with the majority of reviewed studies in which the ratio ranged from 1.1-1.5 (6,7). However, in South Africa, a marginal female predominance has been identified (8). SEER pediatric monograph illustrated that the overall incidence among males was $6.5 \%$ higher compared with that of females (9).

The majority of patients $(75.8 \%)$ were aged $\geq 1$ year at the time of diagnosis, while $24.2 \%$ were aged $<1$ year; the median age was 30 months. This is comparable with the results of a study conducted in Denmark between 1943 and 1980, in which the median age was 29 months (10). A further Danish study, conducted between 1981 and 2000, observed the median age at diagnosis to be 27 months, and $32 \%$ of the patients were $<1$ year of age (11). Additionally, a study of 20 Saudi patients with neuroblastoma reported the median age at onset to be 36 months (12). In a Turkish study, the median age at diagnosis was 43 months, with $21.8 \%$ of patients aged $<1$ year (13), while a study in southern Brazil reported that $25 \%$ of patients were $\leq 1$ year of age, with median age of 33 months at onset (7). Children diagnosed at an age of $<1.5$ years accounted for $63.2 \%$ of a Spanish study population (6). The results of the present study were in discordance with the SEER Pediatric Monograph, which revealed that incidence rate during the second year of life was less than half that of infancy (9). Similarly, Wilson et al (14) reported that young infants, aged $<1$ year, have high incidence of neuroblastoma. This may be attributed to spontaneous regression of neuroblastoma in the younger age group ( $<1$ year) and the lack of diagnosis in primary health care centers, combined with the vagueness of the symptoms, which may lead to a delay in diagnosis (14).

Suprarenal glands were the most common (72.7\%) primary tumor site, which was consistent with the findings of previous studies. SEER monograph reported that, regardless of age, neuroblastomas most frequently occurred in the adrenal gland (9). Moon et al (15) also reported that the adrenal glands were the most common primary site. In addition, the results from a Norwegian cohort indicated that $46.6 \%$ of neuroblastoma masses were identified in the adrenal glands (16). A study of neuroblastoma patients over a period of 10 years in a Saudi hospital reported that the primary sites of involvement included the following: Adrenal, 55\%; retroperitoneal, 15\%; thoracic, $10 \%$, cervical, $5 \%$; pharyngeal, $5 \%$; lumbar, $5 \%$; unknown, 5\% (12). In a study conducted in southern Africa, the most common primary tumor sites included the abdomen (75\%), thorax (15\%), pelvis (5\%), with $5 \%$ located in other sites (8). In addition, a study conducted in southern Brazil over 11 years identified adrenal tumors in $49 \%$ of patients (7). 
In the present study, the majority of patients (76.7\%) had stage IV disease, which is comparable with the results of a Mexican study, in which $88.0 \%$ of patients were classified as stage III, IV, or IVS (17). The findings are also similar to those of Mora et al (2) who found that the majority of neuroblastoma patients presented with advanced, and often unresectable, metastatic disease. Additionally, in southern Brazil, one study reported that $64 \%$ of patients were classified as stage IV (7).

The high percentage of stage IV in the present study may be attributed to a lack of awareness by general practitioners of the probability of cancer, particularly in infancy when localized stages are more common, and to the lack of ultrasound usage, such that tumors are not initially identified, and subsequently regress or are later diagnosed at a more advanced stage in a tertiary hospital. This explanation may also account for the similar findings in Mexico and Brazil $(7,17)$, and is consistent with the postulation by Spix et al (18), in a study of neuroblastoma in Europe between 1978 and 1992, that the variation in stage distribution between countries may be explained by differences in the frequency of diagnosis of localized cases.

Thus, greater consideration of neuroblastoma as a potential diagnosis in primary care centers, and more frequent use of ultrasound, may lead to a higher observed incidence of the disease (due to detection of cases that would otherwise have spontaneously regressed) and a greater proportion of cases of younger patients and of localized disease.

In the present study, $43.8 \%$ of patients exhibited favorable pathology and $56.2 \%$ had unfavorable pathology. These findings were similar to those of a study of Spanish patients in which $39.5 \%$ of neuroblastomas were considered to be of favorable pathology, and $60.5 \%$ of unfavorable pathology (6).

Of the patients in the current study, $60.6 \%$ succumbed to the disease. The estimated survival rate was $30.7 \pm 10.0 \%$, and the mean survival time was $24.2 \pm 5.2$ months. By contrast, a study of Spanish patients reported a mortality rate of $25.8 \%$, with a three-year estimated survival rate of $72.9 \%$ and a mean survival time of 73 months (6). However, $63.2 \%$ of these patients were $<1.5$ years of age and $29.7 \%$ were classified as stage IV, compared with the $24.2 \%$ aged $<1$ year and $76.7 \%$ stage IV in the current cohort. These variations, in addition to differences in medical care level and economic issues, may have contributed to the discrepancies between the findings of the two studies.

In a Mexican study, an overall 5-year survival rate of $64 \%$ was reported (17) whilst in Denmark, the 5-year survival rate showed an increase from 38\% in 1981-1985, to 59\% in 1996-2000 (11). In a cohort study of neuroblastoma in Europe between 1978 and 1992, the 5-year overall survival was $48 \%$ (18). In the present study, the probability of survival was greater for patients of a younger age at onset, with a significantly better outcome for patients $<1$ year of age at presentation; this was consistent with findings from previous studies $(7,19,20)$. SEER monograph revealed that infants aged $<1$ year with neuroblastoma had a more favorable prognosis compared with children $>1$ year of age (9). These findings are consistent across a number of studies, which have reported that an age cut-off of 365 days is clinically significant for risk stratification $(21,22)$. This age cut-off was based on observations by Breslow and McCann over 40 years ago (23).
However, there is conflicting evidence regarding the age cut-off. A number of studies have proposed a higher cut-off of 460 days (24) or 18 months (13). In a Spanish study, patients $<18$ months of age demonstrated better outcomes and a prolonged survival (87 months) compared with those aged $>18$ months. Of the patients who survived, the mean age at diagnosis was 17 months; this was almost half the age of the patients who did not survive (31.3 months) (6).

The association between age and survival is also supported by the results of a large European study, which found that survival was highest in Germany, and that UK mortality rates were significantly higher compared with that of Germany or France. This was due to the higher incidence of the disease below 12 months of age in Germany; in the UK, a deficit of low-stage disease in infants was accompanied by an excess of stage IV disease in older children. Significantly fewer incidental diagnoses were reported in the UK (8\%) compared with Austria (27\%) and Germany (34\%). In Finland, which has the highest survival in the Nordic countries, $>50 \%$ of children with newly diagnosed neuroblastoma were aged $<12$ months, compared with only $33 \%$ of Danish children (25).

Furthermore, age at presentation affects the impact of other prognostic factors on survival due to the genomic amplification of $M Y C N$, which has a profound adverse influence on tumor behavior in infant neuroblastoma patients with metastatic disease. Schmidt et al (26) reported that 3-year event-free survival (EFS) rates were $93 \pm 4 \%$ versus $10 \pm 7 \%$ based on the absence or presence, respectively, of $M Y C N$ amplification in patients $<1$ year of age. By contrast, the adverse prognostic effect of MYCN amplification is diluted in stage IV patients $>1$ year of age at diagnosis.

In the present study, a strong association was observed between the primary tumor site and survival, with $84.2 \%$ of mortalities associated with a suprarenal mass. These results were consistent with that of the European Neuroblastoma Study Group in its study of neuroblastoma in Europe (1982-1992), which revealed that the site of the primary malignancy significantly affected prognosis (27). In two Danish studies, the prognosis was observed to be most favorable in cases involving cervical or thoracic tumors, progressively worsening for thoraco-abdominal and abdominal tumors (other than adrenal), and poorest for adrenal tumors (10,11). Furthermore, the International Neuroblastoma Risk Group Task Force reported that an adrenal primary tumor site was associated with significantly worse EFS compared with all other primary sites combined (28). The effect of the primary malignancy site on survival may be due to the fact that the tumors localized to the neck or thorax result in early symptoms, in contrast to the 'silent' abdominal tumors, which are typically not diagnosed until symptoms from dissemination occur (14).

In the present study, the risk of death from neuroblastoma among females was observed to be 4.9 times higher than that of males, with females accounting for only $22.2 \%$ of survivors. The age at presentation did not differ between males and females, however, 12/14 females (85.7\%) had stage IV disease, in comparison to $11 / 16$ males (68.8\%). Additionally, 5/7 female patients $(71.4 \%)$ exhibited unfavorable pathology, compared with 4/9 male patients (44.4\%).

In a Spanish study (6), $56.3 \%$ of survivors were males. Although this was similar with regard to the improved 
outcome for male patients, the percentages differ markedly from that of the present study, in which only $22.2 \%$ of the survivors were females. Furthermore, in contrast to the current investigation, a number of studies have reported no differences in survival between genders $(10,18,27)$, and a Turkish study found that male gender was a significant determinant of poor prognosis (13).

The pathological status of patients according to the International Neuroblastoma Pathology Classification (INPC) was significantly and strongly correlated with outcome: $77.8 \%$ of patients with unfavorable pathology succumbed to the disease, with a mean survival time of $17.7 \pm 8.9$ months, whilst $71.4 \%$ of those with favorable pathology survived with a mean survival time of $39.7 \pm 8.5$ months. A study by Burgues et al (6) reported that pathology was associated with outcome; the majority of patients $(93.8 \%)$ in the favorable pathology group survived, and the mean survival time for this group (89 months) was significantly higher compared with that of the unfavorable group (57 months). The prognostic value of the INPC system has been confirmed in a number of reports (29).

The findings of the present study revealed that the number of mortalities was significantly increased for the higher disease stages, with a mean survival time of $21.9 \pm 5.6$ months for those with stage IV disease. Similarly, the European Neuroblastoma Study Group reported that disease stage is a highly significant prognostic factor (27). Furthermore, a Spanish study demonstrated that stage IV disease was associated with poor prognosis: In these patients, the a mean survival time was 35 months, and the rate of mortality was $59.3 \%$ (6). Two subsequent studies in Denmark found that clinical stage was a highly significant prognostic factor, with survival rate progressively worsening from stage I to stage IV, and the survival of stage IV-S patients intermediate to these two stages $(10,11)$. In an Iranian study of 43 neuroblastoma patients over 8 years, disease stage was found to be the most important factor predicting outcome (19).

In the current study, biochemical and genetic data were relatively sparse, providing little insight with regard to their impact on long-term outcomes. MYCN status was known for only 24 patients as these were the only cases for which MYCN status had been requested. This was attributed to the fact that, from 2005-2006, the chemotherapy protocol used for neuroblastoma was the same for all patients [PE-CADO protocol (30)] since data regarding different risk factors was limited and risk-based treatments had not yet been established, and $M Y C N$ status did not affect the treatment strategy. Even after application of the risk stratification system in treatment, the hospital assessed MYCN status only in patients for whom this would determine risk classification. Accordingly, 5 out of 6 patients for whom MYCN status was assessed had favorable pathology. These restrictions on the determination of $M Y C N$ status are attributed to the cost of such assessment and to limited resources. This was also observed in a Turkish study (9), and in a study in southern Africa (8), which proposed that serum LDH, serum ferritin and histology may be utilized as simple and inexpensive tests, with good predictive values for outcome, in countries with limited resources with which to stratify patients due to the unavailability of molecular tests. This is used in many developing countries where detailed molecular or biological tests remain unavailable. Tailored therapy is primarily based on clinical factors including age, disease stage and pathology. Consistently, the results of the present study revealed that clinical data were valuable and useful in the risk stratification of patients when other biological and molecular determinants were not available. However, previous studies support the association between adverse outcomes and MYCN amplification (31).

The results of the present study revealed that risk-based chemotherapy protocols did not improve cure rates or survival compared with the previously used PE-CADO protocol. The limited supportive care and financial resources in Egypt, as in other developing countries, hinders the application of aggressive chemotherapy followed by bone marrow transplantation (8).

In the current study, suprarenal primary tumor sites were significantly associated with an age of $\geq 1$ year at presentation. This is consistent with a Turkish study, in which thoracic and neck tumors were more prevalent in patients aged $<18$ months, and abdominal disease was more prevalent in patients aged $>18$ months (13). This may be due to the in utero initiation of neuroblastoma, meaning that tumors originating in hidden sites, including suprarenal sites, take a greater period of time to present, and are therefore associated with the older age group, in contrast to tumors originating in sites such as the neck and paraspinal regions, where they are detectable at an earlier stage.

The present study revealed a statistically significant association between age at presentation and INSS stage, with older age associated with higher stages, and a significant cut-off age of 12 months. This finding is concordant with previous studies in which biological evidence of a correlation between age and stage was identified (13,32). However, a Mexican study found no association between disease stage and age at the time of diagnosis (17).

Only $42.9 \%$ of patients who presented at $<1$ year of age had localized disease (stages I, II, III). This may be attributed to the lack of diagnosis of localized stages in infants, leading to the appearance of an aberrantly high percentage of metastatic stages in this age group. This hypothesis is supported by the results of a German study in which infants were screened, and $87 \%$ of the cases of the disease were found to be localized (stages I, II or III) (33). The present study revealed that progression in stage implied a higher proportion of cases with unfavorable pathology, reaching $60.0 \%$ in INSS stage IV tumors. This was consistent with the results of a Spanish study (6), which reported that tumor stage was significantly associated with INPC prognosis, with unfavorable cases reaching $89.3 \%$ in INSS stage IV tumors. However, in a Mexican study (17), no correlation between Shimada classification (favorable or unfavorable histology) and stage was identified.

In the present study, the higher percentage of patients with evidence of central nervous system metastasis in the unfavorable pathology group (85.7\%), compared with the favorable pathology group (55.6\%), may account for the association between pathology and stage.

The present study identified age to be significantly associated with pathology in continuous manner, however, this was more clinically and statistically significant when an age cutoff of 12 months was used to group patients. This was consistent with the findings of London et al (32) and of Shimada (34), who observed biological evidence of a correlation between age and histopathology. The association between age at presentation, 
disease stage and pathology supports the hypothesis that neuroblastoma consists of more than two distinct subtypes (35).

In conclusion, the association between age at presentation, stage and pathology supports the hypothesis that neuroblastoma is a heterogeneous disease. The establishment of an effective, computerized, research-oriented registration system for neuroblastoma is urgently required in north east Egypt. Furthermore, larger international multicenter studies are required to support the findings of the present study.

\section{References}

1. Schwab M,WestermannF,Hero B and BertholdF: Neuroblastoma: Biology and molecular and chromosomal pathology. Lancet Oncol 4: 472-480, 2003.

2. Mora J, Gerald WL, Qin J and Cheung NK: Evolving significance of prognostic markers associated with treatment improvement in patients with stage 4 neuroblastoma. Cancer 94: 2756-2765, 2002

3. De Bernardi B, Mosseri V, Rubie H, et al; SIOP Europe Neuroblastoma Group: Treatment of localised resectable neuroblastoma. Results of the LNESG1 study by the SIOP Europe Neuroblastoma Group. Br J Cancer 99: 1027-1033, 2008.

4. Look AT, Hayes FA, Nitschke R, et al: Cellular DNA content as a predictor of response to chemotherapy in infants with unresectable neuroblastoma. N Engl J Med 311: 231-235, 1984.

5. Matthay KK, Reynolds CP, Seeger RC, et al: Long-term results for children with high-risk neuroblastoma treated on a randomized trial of myeloablative therapy followed by 13-cis-retinoic acid: A children's oncology group study. J Clin Oncol 27: 1007-1013, 2009.

6. Burgues O, Navarro S, Noguera R, Pellín A, Ruiz A, Castel V and Llombart-Bosch A: Prognostic value of the International Neuroblastoma Pathology Classification in Neuroblastoma (Schwannian stroma-poor) and comparison with other prognostic factors: A study of 182 cases from the Spanish Neuroblastoma Registry. Virchows Arch 449: 410-420, 2006.

7. Parise IZ, Haddad BR, Cavalli LR, et al: Neuroblastoma in southern Brazil: An 11-year study. J Pediatr Hematol Oncol 28 $82-87,2006$

8. Hesseling PB, Ankone K, Wessels G, Schneider JW, Du Plessis L and Moore S: Neuroblastoma in southern Africa: Epidemiological features, prognostic factors and outcome. Ann Trop Paediatr 19: 357-363, 1999.

9. Goodman MT, Gurney JG, Smith MA, et al: Sympathetic nervous system tumors. In: Cancer incidence and survival among children and adolescents: United States SEER Program 1975-1995. National Cancer Institute, SEER Program. NIH Pub. No.99-4649. Ries LAG, Smith MA, Gurney JG, et al (eds). Bethesda, MD, 1999.

10. Carlsen NLT, Christensen IJ, Schroeder H, Bro PV, Erichsen G Hamborg-Pedersen B, Jensen KB and Nielsen OH: Prognostic factors in neuroblastomas treated in Denmark from 1943 to 1980. A statistical estimate of prognosis based on 253 cases. Cancer 58 : 2726-2735, 1986.

11. Schroeder H, Wacher J, Larsson H, Rosthoej S, Rechnitzer C, Petersen BL and Carlsen NL: Unchanged incidence and increased survival in children with neuroblastoma in Denmark 1981-2000: A population-based study. Br J Cancer 100: 853-857, 2009.

12. al-Mulhim I: Neuroblastoma in children: A 10-year experience in Saudi Arabia. J Trop Pediatr 44: 77-80, 1998.

13. Aydn GB, Kutluk MT, Yalçn B, Büyükpamukçu M, Kale G, Varan A, Akyüz C, Senocak ME and Büyükpamukçu N: Neuroblastoma in Turkish children: Experience of a single center. J Pediatr Hematol Oncol 31: 471-480, 2009.

14. Wilson LM and Draper GJ: Neuroblastoma, its natural history and prognosis: A study of 487 cases. BMJ 3: 301-307, 1974.

15. Moon SB, Park KW, Jung SE and Youn WJ: Neuroblastoma: Treatment outcome after incomplete resection of primary tumors. Pediatr Surg Int 25: 789-793, 2009.

16. Méhes G, Luegmayr A, Kornmüller R, Ambros IM, Ladenstein R, Gadner H and Ambros PF: Detection of disseminated tumor cells in neuroblastoma: 3 log improvement in sensitivity by automatic immunofluorescence plus FISH (AIPF) analysis compared with classical bone marrow cytology. Am J Pathol 163: 393-399, 2003.
17. Juárez-Ocaña S, Palma-Padilla V, González-Miranda G, Siordia-Reyes AG, López-Aguilar E, Aguilar-Martínez M, Mejía-Aranguré JM, Carreón-Cruz R, Rendón-Macías ME and Fajardo-Gutiérrez A: Epidemiological and some clinical characteristics of neuroblastoma in Mexican children (1996-2005). BMC Cancer 9: 266, 2009.

18. Spix C, Aareleid T, Stiller C, Magnani C, Kaatsch P and Michaelis J: Survival of children with neuroblastoma. time trends and regional differences in Europe, 1978--1992. Eur J Cancer 37: 722-729, 2001

19. Arzanian MT and Esfahani H: Neuroblastoma; variable symptoms of a neurogenic tumor; a report from Iran. IJCN 3: 33-39, 2008.

20. Grosfeld JL: Risk-based management of solid tumors in children. Am J Surg 180: 322-327, 2000.

21. Joshi VV, Cantor AB, Altshuler G, et al: Age-linked prognostic categorization based on a new histologic grading system of neuroblastomas. A clinicopathologic study of 211 cases from the Pediatric Oncology Group. Cancer 69: 2197-2211, 1992.

22. Berthold F, Kassenböhmer R and Zieschang J: Multivariate evaluation of prognostic factors in localized neuroblastoma. Am J Pediatr Hematol Oncol 16: 107-115, 1994.

23. Breslow N and McCann B: Statistical estimation of prognosis for children with neuroblastoma. Cancer Res 31: 2098-2103, 1971.

24. Saito T, Tsunematsu Y, Saeki M, Honna T, Masaki E, Kojima Y and Miyauchi J: Trends of survival in neuroblastoma and independent risk factors for survival at a single institution. Med Pediatr Oncol 29: 197-205, 1997.

25. Powell JE, Estève J, Mann JR, Parker L, Frappaz D, Michaelis J, Kerbl R, Mutz ID and Stiller CA: Neuroblastoma in Europe: Differences in the pattern of disease in the UK. SENSE. Study group for the Evaluation of Neuroblastoma Screening in Europe. Lancet 352: 682-687, 1998

26. Schmidt ML, Lukens JN, Seeger RC, et al: Biologic factors determine prognosis in infants with stage IV c. J Clin Oncol 18: 1260-1268, 2000.

27. Cotterill SJ, Pearson AD, Pritchard J, Foot AB, Roald B, Kohler JA and Imeson J: Clinical prognostic factors in 1277 patients with neuroblastoma: Results of The European Neuroblastoma Study Group 'Survey' 1982-1992. Eur J Cancer 36: 901-908, 2000.

28. Cohn SL, Pearson AD, London WB, et al; INRG Task Force: The International Neuroblastoma Risk Group (INRG) classification system: An INRG Task Force report. J Clin Oncol 27: 289-297, 2009

29. Matthay KK, Perez C, Seeger RC, et al: Successful treatment of stage III neuroblastoma based on prospective biologic staging: A Children's Cancer Group study. J Clin Oncol 16: 1256-1264, 1998

30. Bernard JL,Philip T,Zucker JM, et al: Sequential cisplatin/VM26 and vincristine/cyclophosphamide/doxorubicin in metastatic neuroblastoma: An effective alternating non-cross-resistant regimen? J Clin Oncol 5: 1952-1959, 1987.

31. Bagatell R, Rumcheva P, London WB, et al: Outcomes of children with intermediate-risk neuroblastoma after treatment stratified by MYCN status and tumor cell ploidy. J Clin Oncol 23: 8819-8827, 2005.

32. London WB, Castleberry RP, Matthay KK, et al: Evidence for an age cutoff greater than 365 days for neuroblastoma risk group stratification in the Children's Oncology Group. J Clin Oncol 23: 6459-6465, 2005.

33. Schilling FH, Spix C, Berthold F, et al: Neuroblastoma screening at one year of age. N Engl J Med 346: 1047-1053, 2002.

34. Shimada H, Umehara S, Monobe Y, et al: International neuroblastoma pathology classification for prognostic evaluation of patients with peripheral neuroblastic tumors: A report from the Children's Cancer Group. Cancer 92: 2451-2461, 2001.

35. Pritchard J and Hickman JA: Why does stage 4s neuroblastoma regress spontaneously. Lancet 344: 869-870, 1994. 